\title{
Ergodic Properties of an Infinite One Dimensional Hard Rod System ${ }^{\star}$
}

\author{
Michael Aizenman \\ Belfer Graduate School of Science, Yeshiva University, New York, N.Y., USA \\ Sheldon Goldstein $\star \star$ \\ Institute for Advanced Study, Princeton, New Jersey, USA \\ Joel L. Lebowitz \\ Belfer Graduate School of Science, Yeshiva University, New York, N.Y., USA
}

Received June 15, 1974

\begin{abstract}
It is shown that an infinite one dimensional system of hard rods for which the "effective" velocities of the pulses (free velocity plus a drift term due to collisions) are bounded away from some neighborhood of 0 is Bernoulli. This generalizes a result of Sinai who showed that some hard rod systems are $K$-systems.
\end{abstract}

\section{Introduction}

Since the number of particles contained in a typical macroscopic system is very large $\left(\sim 10^{26}\right)$ there is great interest from the point of view of statistical mechanics in the ergodic properties of infinite systems (corresponding to the thermodynamic limit in equilibrium statistical mechanics). In this note we extend the results of Sinai [1] who showed that a one dimensional system of hard rods is a $K$-system. We prove, under slightly restrictive conditions on the velocity distribution, that this system is Bernoulli. We also clear up some points in Sinai's proof.

Before describing our results we would like to point out that care must be exercised in drawing analogies between the ergodic properties of finite and infinite systems, as the dependence of these properties on the interactions between the particles, and thus also their physical interpretation, may be very different in the two cases. Thus, while a finite ideal gas (classical system of non-interacting point particles) is not even ergodic the infinite ideal gas has the strongest possible ergodic properties: it is a Bernoulli system [2]. This "good" ergodic behavior of the infinite ideal gas does not, unfortunately; provide any

$\star$ Research sponsored in part by AFOSR Grant No. 73-2430A.

$\star \star$ Research sponsored in part by The National Science Foundation Grant No. GP-16147A No. 1. 
guarantee of similar good ergodic behavior of infinite systems of interacting particles.

The explanation of the good ergodic properties of the infinite ideal gas in simple: local disturbances "fly off" unhindered to infinity where they are no longer observable (with respect to measures natural from the point of view of statistical mechanics, i.e., measures concentrated on local observables [3]). Formally the proof for the infinite ideal gas is obtained by showing that the flow is isomorphic to the process obtained by observing the particles which at any moment cross a given hyperplane (a point for one dimensional systems). The absence of interactions plays here a double role:

1) The fact that the "information" "flows" unperturbed guarantees that all of it eventually gets recorded by the local observations (on the hyperplane), in a way which enables one to reconstruct the phase-space description at the time $t=0$.

2) The observed "information" does not return to the hyperplane, making observations at different times independent.

It is plausible that some infinite systems of interacting particles will no longer admit a generating "local observation". This would not rule out strong ergodic properties; their proof however would require different methods which may, in fact, lead to stronger results [4]. In those systems which do admit representation by a process constructed on local observations, the interaction will induce a dependence among the observations at different times, possibly preventing strong ergodic properties (of the local observation).

It is with an eye to understanding the latter behavior that we consider the effect of a hard core interaction in an infinite one dimensional gas. The motion of a "velocity pulse" in this system is a combination of a steady flow with discrete independent jumps back and forth. Pulses with effective velocity 0 would reappear infinitely often at any place on the line. It is shown that an infinite system of hard rods for which the effective velocities are bounded away from some neighborhood of 0 is Bernoulli.

\section{Description of the System and the Main Result}

Let $X$ denote the phase space of an infinite system of hard rods of diameter $d>0 . x \in X$ is a countable collection of pairs $x=\left\{\left(x_{\alpha}, v_{\alpha}\right)\right\}_{\alpha}$, where $x_{\alpha}$ is the position of the left corner (or any other fixed point on it) of a rod and $v_{\alpha}$ its velocity. Let $\mu$ denote the translationally invariant measure on $X$ under which:

1) The free distances between consecutive rods (given that the origin is covered) are jointly independent and identically distributed, 
with an exponential distribution of parameter $\varrho>0$. $\varrho=n /(1-$ nd) where $n$ is the average particle density.

2) The velocities are independent and identically distributed with probability measure $v$, which has a finite first moment.

Let $S_{t}$ denote the flow on $X$ under which each particle in $x \in X$ moves freely $\left(\frac{d x_{\alpha}}{d t}=v_{\alpha}, \frac{d v_{\alpha}}{d t}=0\right)$ except for elastic collisions. By an argument similar to Sinai's [1] (used for systems with a Maxwellian velocity distribution) it may be shown that $\left\{S_{t}\right\}$ is well defined on a set of full measure.

For convenience, reference will be made to velocity pulses, whose positions are the positions of rods but which are understood to exchange rods in a collision. A pulse of velocity $v$ moves at this velocity except for moments of collision, when it jumps the distance $d$ in the direction of the other colliding particle. However, the "free distance" between two pulses (obtained by subtracting the total length of rods between them) behaves linearly in time.

Moreover, for a given position of a pulse, the "free distances" to other pulses are distributed along the line with a Poisson distribution. Therefor, for given velocity and position of a pulse, the collisions it undergoes at different times are independent.

Lemma (2.1). There is a measurable set $\tilde{X} \subset X, \mu(\tilde{X})=1$, such that $\forall x \in \tilde{X}$ the following holds; let $v$ be the velocity of a pulse in $x$, then:

1) During the motion induced on it by $\left\{S_{t} x\right\}$, the pulse crosses the origin.

2) The induced average velocity of the pulse for the time interval $[0, t]$, approaches

$$
v_{\mathrm{eff}}(v)=v+\varrho d(v-E(v)), \quad \text { as } \quad t \rightarrow \pm \infty .
$$

The lemma can be proven by showing that Properties 1) and 2) hold, with probability 1 , for each pulse separately (labeling each pulse by $\left.l_{\alpha}=\left[x_{\alpha} / d\right]\right)$. This can be easily done with the help of the previous remarks.

Note that for pulses of velocity $v \neq 0$ 1) follows from 2), while for pulses of velocity $v=0$ 1) holds as a result of the fluctuations in the number of collisions.

Let $v_{0}=\frac{\varrho d}{1+\varrho d} E(v)$. By Lemma (2.1), pulses of velocity $v_{0}$ propagate with the effective velocity (for long times) 0 . Since the effective velocity contains a part (of positive variance) which is due to independent collisions, pulses of this velocity recur infinitly often at any place on the line. 
With the help of Lemma (2.1) one can generalize Sinai's result [1] to obtain

Theorem (2.2) $(X, \mu, S)^{1} \quad(d>0)$ is a K-system for any velocity probability distribution $v$.

The proof will not be given here. Let us remark however that Sinai's proof carries over to systems with $v\left(v=v_{0}\right)=0$. Other systems are covered by a modified argument.

Our main result is

Theorem (2.3). The dynamical system $\left(X, \mu, S_{t}\right)$ for which the velocity distribution $v$ satisfies

$$
v\left(\left|v-v_{0}\right|<\delta\right)=0
$$

for some $\delta>0$ and $v_{0}=\frac{\varrho d}{1+\varrho d} E(v)$, is a Bernoulli flow.

\section{Reduced Description}

As already indicated, we are interested in a representation of the system by a process generated by local observations. However, since collisions occur at any interval on the line, the reappearance of a pulse at the origin depends on the distribution of particles elsewhere, inducing a complicated dependence among local observations taken at different times. In the following "reduced description" (which is limited to one dimensional systems) the discontinuities in the trajectory of a pulse result from effects which take place at the origin.

For any $x \in X$, let us label the particles so that their positions at time $t=0^{-}$satisfy

$$
\ldots x_{-2}<x_{-1}<0 \leqq x_{0}<x_{1} \ldots
$$

taking the limit $t \uparrow 0$ for each inequality separately.

Definition (3.1). Let $\left(x_{i}, v_{i}\right)$ be the position and velocity of the $i$-th particle in $x \in X$. Its reduced position is given by $q_{i}=x_{i}-\mathrm{id}$.

Clearly, an equivalent description of $x \in X$ is given by $y=\left\{\left(q_{i}, v_{i}\right)\right\}$, the enumerated collection of the reduced positions and velocities of the induced particles. Note that the unenumerated collection $\left\{\left(q_{\alpha}, v_{\alpha}\right)\right\}$ may not determine $x \in X$ uniquely.

Definition (3.2). The reduced phase space, $Y$, is the class of enumerated collections $y=\left\{\left(q_{i}, v_{i}\right)\right\}_{i \in \mathbb{Z}}$ for which

1) $q_{i} \leqq q_{i+1} \quad \forall i \in \mathbb{Z}$.

2) $0 \leqq q_{0}$.

\footnotetext{
1 We will write $S$ in place of $S_{1}$.
} 
3) $q_{-1} \leqq d$.

4) The replacement $q_{i} \rightarrow q_{i}+t v_{i}, t=0^{-}$, makes each of the above inequalities strict (except if $v_{i}=v_{i+1}$ or $v_{0}=0$ ).

The mapping $\varphi: X \rightarrow Y$ which carries $x=\left\{\left(x_{\alpha}, v_{\alpha}\right)\right\}$ to the enumerated collection of the reduced positions and velocities of its particles, ordered as above, is $1-1$ and onto.

By a harmless abuse of notation, let $\mu$ and $S_{t}$ denote the measure and the flow induced on $Y$ by the correspondence $\varphi$.

Lemma (3.3). With respect to the measure $\mu$, the distribution of the reduced positions and velocities (ignoring the labeling) of those pulses of $y \in Y$ which lie in $\mathbb{R} \backslash[0, d]$ is isomorphic to the Poisson distribution of points, with density $\varrho$, over $((\mathbb{R} \backslash[0, d], l) \otimes(\mathbb{R}, v)$ (l being the Lebesgue measure) and is independent of the distribution and labeling of pulses in $[0, d]$.

$$
\text { Proof. Let } \left.\underset{(-)}{q_{+}}={\underset{(\max )}{\min }}_{q_{i}} \mid \begin{array}{c}
q_{i}>d \\
(<0)
\end{array}\right\} \text {. }
$$

The lemma follows from the observation that the distributions of both $q_{+}-d$ and $\left|q_{-}\right|$are exponential with parameter $\varrho$, independent of the configuration in $[0, d]$ and of each other.

We remark that $\mu$ has the following realization: Let $\bar{Y}=Y_{+} \otimes Y_{-}$ be the product of two independent Poisson distributions of points with density $\varrho, Y_{+}$over $([0, \infty), l) \otimes(\mathbb{R}, v)$, whose left particle is labeled " 0 "; and $Y_{-}$over $((-\infty, d], l) \otimes(\mathbb{R}, v)$, whose right particle is labeled " -1 ". $(Y, \mu)$ is isomorphic to $\left(\bar{Y}^{\prime}, \mu^{\prime}\right)$, where $\bar{Y}^{\prime}=\left\{y \in \bar{Y} \mid q_{0} \geqq q_{-1}\right\}$ and $\mu^{\prime}$ is the induced probability measure on $\bar{Y}^{\prime}$.

Let $y \in Y$. We denote by $N(t, y)$ the directed number of crossings of the origin by particles in $x=\varphi^{-1}(y)$, during the time interval $[0, t)$, counting crossings from left (right) as positive (negative). $K(y)$ will denote the index of the first particle whose reduced position (in $S_{0}-y$ ) is non negative.

Consider now the motion of the pulses in $y \in Y$ induced by the flow $S_{t}$. The pulses move at their characteristic velocity, exchanging indices at collisions, except for moments at which a particle crosses the origin (in $x$ ), when the reduced positions of all the pulses are shifted by $\mp d$ and their index values change by \pm 1 , depending on the direction of the crossing. The reduced distance traveled by a pulse $(q, v) \in y \in Y$ during the time interval $[0,1)$ is thus equal to $t v-d N(t, y)$.

Lemma (3.4). For almost any $y \in Y$

$$
\lim _{t \rightarrow \infty} \frac{N(t, y)}{t}=\frac{\varrho}{1+\varrho d} E(v), \quad \text { for } t \in \mathbb{Z} .
$$


Proof. Since our system is ergodic, Theorem (2.2), it follows that $N(t, y) / t=1 / t \sum_{i=0}^{t-1} N\left(1, S_{i} y\right) \underset{(t \rightarrow \infty)}{\longrightarrow} E(N(1, y))$ almost surely (a.s.) .

To compute $E(N(1, y))$ we observe that the average velocity, in the reduced description, of a pulse of velocity $v$ is

$$
[t v-d N(t, y)] / t \underset{(t \rightarrow \infty)}{\longrightarrow} v-d E(N(1, y)),
$$

which corresponds to velocity $[v-d E(N(1, \cdot))] \cdot(1+\varrho d)$ in terms of "real" distance. Comparing the above with Lemma (2.1) we obtain

$$
E(N(1, \cdot))=\frac{\varrho}{1+\varrho d} E(v) .
$$

\section{Process Description}

Definition (4.1). We will call a pulse $(q, v) \in y \in Y$ marked at time $t$ if one of the following holds

a) $0 \leqq q+t v-d N(t, y) \leqq d$.

b) $q+t v-d N(t, y)<0$ and $q+(t+1) v-d N(t+1, y) \geqq 0$.

c) $q+t v-d N(t, y)>d$ and $q+(t+1) v-d N(t+1, y) \leqq d$.

We now define a partition of the phase space which will be used to represent our system by a process. In order to apply approximation techniques coarser partitions will be defined as well.

Definition (4.2).

1) Denote by $\eta$ the partition of $Y$ generated by:

a) $N(1, y)$

b) $K(y)$, and

c) the reduced positions and velocities of those pulses of $y$ which are marked at the time $t=0$.

2) Denote by $\eta^{(k)}(\forall k \in \mathbb{Z})$ the partition whose typical element $C^{(k)}(\bar{y}) \in \eta^{(k)}$ is the collection of the phase space points $y \in Y$ for which:

a) $N(1, y)=N(1, \bar{y})$

b) $K(y)=K(\vec{y})$, and

c) there is a $1-1$ correspondence between the pulses marked at the time $t=0$ in $y$ and $\bar{y}$, such that at the times $t=0,1$ the corresponding pulses are at the same distances from the origin, measured in intervals of the size $d / 2^{k}$.

In essence, $\eta$ partitions $Y$ (and therefore $X$ ) by the characteristics of the pulses which in the reduced description appear in the interval $[0, d]$ during the time $[0,1]$, disregarding those which do appear but eventually cross back. The pulses have to be observed in an interval since their trajectories have discontinuities of the size $d$. 
It is an advantage of the reduced description that $\eta(x)$ contains the full information about those pulses of $x$ which are in a certain region [which depends on $\eta(x)$ ] of the one particle phase space, and is independent of the characteristics of the pulses elsewhere. To show this we need the following lemma.

Denote by $\alpha^{ \pm}(n, t)$ the regions in the one particle phase space, defined by

$$
\alpha^{( \pm)}(n, t)=\left\{\begin{array}{ll}
(q, v) & \begin{array}{l}
q \leqq 0, q+v t-d n>0 \\
(\geqq)
\end{array}
\end{array}\right\} .
$$

Lemma 4.3. Let $y \in Y$ and let $m=N(t, y)$. If $y$ and $\bar{y} \in Y$ possess the same occupation numbers for pulses in the regions $\alpha^{ \pm}(m, t)$ and $\alpha^{ \pm}(m+1, t)$, and $K(y)=K(\bar{y})$ then $N(t, \bar{y})=m$.

Proof. Denote by $\hat{N}_{y}(\beta)$ the number of pulses in $y \in Y$ which occupy a given region $\beta$ in the one particle phase space.

Notice that for $\left.m=N(t, y), \alpha^{\ddagger}\right)(m, t)$ is the region of those pulses whose reduced positions change from non-positive (non-negative) in $y$ to positive (negative) in $S_{t} y$.

Keeping in mind the fact that the index values get readjusted each time a particle (in the unreduced description) crosses the origin one obtains

therefore

$$
K\left(S_{t} y\right)=K(y)-\hat{N}_{y}\left(\alpha^{+}(m, t)\right)+\hat{N}_{y}\left(\alpha^{-}(m, t)\right)+m,
$$

$$
K(y)-\hat{N}_{y}\left(\alpha^{+}(m, t)\right)+\hat{N}_{y}\left(\alpha^{-}(m, t)\right)+m \leqq 0 .
$$

Similarly, $K(y)-\hat{N}_{y}\left(\alpha^{+}(m+1, t)\right)+\hat{N}_{y}\left(\alpha^{-}(m+1, t)\right)+m$ is the index of the first pulse in $S_{t-0} y$ whose reduced position is not smaller than $d$, therefore

$$
K(y)-\hat{N}_{y}\left(\alpha^{+}(m+1, t)\right)+\hat{N}_{y}\left(\alpha^{-}(m+1, t)\right)+(m+1)>0 .
$$

Consider now the function (defined on $\mathbb{Z}$ )

$$
f_{y}(n)=K(y)-\hat{N}_{y}\left(\alpha^{+}(n, t)\right)+\hat{N}_{y}\left(\alpha^{-}(n, t)\right)+n .
$$

Since $\alpha^{+}(n, t) \supset \alpha^{+}(n+1, t)$ and $\alpha^{-}(n, t) \subset \alpha^{-}(n+1, t), f(n)$ is strictly increasing. Moreover, by the above inequalities, $N(t, y)$ is the unique solution of

$$
f_{y}(n) \leqq 0, \quad f_{y}(n+1)>0 .
$$

Now, by the conditions of the lemma,

$$
f_{\bar{y}}(m)=f_{y}(m) \leqq 0 \quad \text { and } \quad f_{\bar{y}}(m+1)=f_{y}(m+1)>0,
$$

implying $N(t, y)=N(t, \bar{y})$.

Corollary (4.4). Let the region $\alpha(n)$ of the one particle phase space be given by

$$
\alpha(n)=\alpha^{+}(n, 1) \cup \alpha^{-}(n+1,1) \cup([0, d] \otimes \mathbb{R}) .
$$


If $N(1, y)=n$, then $\eta^{k}(y)$ is independent of the characterietics of those pulses which occupy $\alpha(n)^{c}$ (the complement of $\alpha(n)$ ). Notice that $\eta(y)$ contains the full information regarding the pulses in $\alpha(n)$.

The above independence will be used to establish strong ergodic properties for the process defined by $\eta^{(k)}$.

\section{Tools Used}

In proving the Bernoulli property we will make use of the method developed by Ornstein which utilizes the following results [5]:

Lemma (5.1). The dynamical system $\left(X, \mu,\left\{T_{t}\right\}\right)$ is Bernoulli if $\left(X, \mu, T_{1}\right)$ is a Bernoulli shift.

Lemma (5.2). If $A_{1} \subset A_{2} \subset A_{3} \subset \ldots$ form an increasing sequence of $T$-invariant $\sigma$-algebras, if $\bigvee_{0}^{\infty} A_{k}=B$, and if for each $n,\left(X, A_{n}, \mu, T\right)$ is a Bernoulli shift then $(X, B, \mu, T)$ is a Bernoulli shift.

This lemma enables one to use results obtained for processes defined by countable partitions. Given a generating partition $P$, the dynamical system $(X, \mu, T)$ is isomorphic to the process $(T, P)$ with the induced measure.

Definition (5.3). The partition $P=\left\{P_{i}\right\}$ is $\varepsilon$-independent of $Q=\left\{Q_{j}\right\}$ if there is a class $C$ of sets in $Q$ such that

a) $\mu(\cup C) \geqq 1-\varepsilon$

b) $\sum_{i}\left|\mu\left(P_{i} \mid Q_{j}\right)-\mu\left(P_{i}\right)\right| \leqq \varepsilon \quad \forall Q_{j} \in C$.

Definition (5.4). A partition $P$ is called weakly-Bernoulli for an automorphism $T$ if given $\varepsilon>0$ there exists an $N$ such that for all $m \geqq 1$ :

$$
\bigvee_{-(N+m)}^{-N} T^{i} P \text { is } \varepsilon \text {-independent of } \bigvee_{0}^{m} T^{i} P
$$

Lemma (5.5). If the partition $P$ is weakly-Bernoulli for the automorphism $T$ then $(T, P)$ is a Bernoulli process.

\section{Proof of the Main Result}

Let us restate the main Theorem(2.3), using the notation of the previous sections.

Theorem (6.1). The dynamical system $\left(Y, \mu, S_{t}\right)$ for which

$$
v\left(\left|v-v_{0}\right|<\delta\right)=0, \text { for some } \delta>0 \text { and } v_{0}=\frac{\varrho d}{1+\varrho d} E(v),
$$

is a Bernoulli system. 
The proof will consist of several steps. The "times" $t$ mentioned throughout this section are to be understood as integral.

Lemma (6.2). Let the "good" sets be given by

$$
G_{t}^{( \pm)}=\left\{y \in Y \mid \sup _{\substack{t^{\prime}>t \\\left(t^{\prime}<-t\right)}}\left\{\left(\left|d N\left(t^{\prime}, y\right)-t^{\prime} v_{0}\right|+d\right) /\left|t^{\prime}\right|\right\}<\delta\right\} .
$$

Then $\forall \varepsilon>0, \exists t_{1}(\varepsilon, \delta)>0$ for which $\mu\left(G_{t}^{+}\right), \mu\left(G_{t}^{-}\right)>1-\frac{(\varepsilon)^{2}}{10}$.

Proof. By Lemma (3.4) $\lim _{|t| \rightarrow \infty} d N(t, y) / t=v_{0}$ for almost every $y \in Y$, therefore $\sup _{\left|t^{\prime}\right|>t}\left\{\left(\left|d N\left(t^{\prime}, y\right)-t^{\prime} v_{0}\right|+d\right) / t^{\prime}\right\} \underset{(t-\infty)}{\longrightarrow} 0$ a.s implying convergence in probability, which is Lemma (6.2).

For the system under consideration $v\left(\left|v-v_{0}\right|<\delta\right)=0$; let us assume therefore that there are no pulses of velocity $v,\left|v-v_{0}\right|<\delta$ (in fact, we are confining the discussion to a subset of $Y$ of full measure).

Let $G_{t}=G_{t}^{+} \cap G_{t}^{-}$.

Remark (6.3). $\forall y \in G_{t}$, no pulse of $y$ whose reduced position was in $[0, d]$ at a time $t^{\prime} \in(-\infty,-t]$ will reappear in $[0, d]$ at a time $t^{\prime \prime} \in[t, \infty)$ or at $t^{\prime \prime}=0^{2}$. This can be easily shown, remembering that the reduced distance traveled by a pulse of velocity $v$ between the times $t^{\prime}$ to $t^{\prime \prime}$ is

$$
v\left(t^{\prime \prime}-t^{\prime}\right)-d\left(N\left(t^{\prime \prime}, y\right)-N\left(t^{\prime}, y\right)\right) .
$$

Definition (6.4). Denote by $\zeta$ the partition generated by

1) $K(y)$,

2) the number of velocity pulses in $[0, d]$ for which $v>v_{0}+\delta$ and the number of those for which $v<v_{0}-\delta$.

We now come to the key step in the argument:

Lemma (6.5). Let a be a set measurable with respect to $\bigvee_{-(t+1)}^{\infty} S^{i} \eta(t>0)$, $b$ measurable with respect to $\bigvee_{-\infty}^{-(t+1)} S^{i} \eta$, and $\zeta_{k} \in \zeta$. Then

$$
\mu\left(a \mid b, \zeta_{k}, G_{t}\right)=\mu\left(a \mid \zeta_{k}, G_{t}\right) .
$$

Proof. Note that [by virtue of Corollary (4.4) and Lemma (4.3)] $a \cap G_{t}^{+}$and $b \cap G_{t}^{-}$depend on the distribution of pulses in two corresponding regions in the one particle phase space, whose intersection (after taking out the slice $\mathbb{R} \otimes\left(v_{0}-\delta, v_{0}+\delta\right)$ ) is $[0, d] \otimes \mathbb{R}$. Further, the dependence of $a \cap G_{t}^{+}$and of $b \cap G_{t}^{-}$on the distribution of pulses in $[0, d] \otimes \mathbb{R}$ is only through the variables used to define the partition $\zeta$ [Definition (6.4)]. Therefore, by Lemma (3.3), on a given element $\zeta_{k} \in \zeta, a \cap G_{t}^{+}$and $b \cap G_{t}^{-}$are independent:

$$
\text { a) } \mu\left(a \cap G_{t}^{+} \mid \zeta_{k}, b \cap G_{t}^{-}\right)=\mu\left(a \cap G_{t}^{+} \mid \zeta_{k}\right)
$$

\footnotetext{
2 Here the times are not to be understood as necessarily integral.
} 
In particular, by choosing $b=Y$ and then $a=Y$, we obtain

and

b) $\mu\left(a \cap G_{t}^{+} \mid \zeta_{k}, G_{t}^{-}\right)=\mu\left(a \cap G_{y}^{+} \mid \zeta_{k}\right)$,

$$
\text { c) } \quad \mu\left(G_{t}^{+} \mid \zeta_{k}, G_{t}^{-}\right)=\mu\left(G_{t}^{+} \mid \zeta_{k}\right) \text {. }
$$

The lemma follows now from a), b), and c).

We observe therefore that our system exhibits an approximate Markov property. The proof that it is Bernoulli will follow in a way similar to a proof that a $K$ Markov system is Bernoulli (actually, for this end, mixing could replace the $K$ property). We thus first consider the space $Y$ equipped with the $\sigma$-algebras $\bigvee_{i=-\infty}^{\infty} S^{i} \eta^{(k)}$.

Theorem (6.6). For any integer $k$, the dynamical system

$$
\left(Y, \bigvee_{-\infty}^{\infty} S^{i} \eta^{(k)}, \mu, S\right)
$$

for which the velocity distribution $v$ satisfies

$$
v\left(\left|v-v_{0}\right|<\delta\right)=0, \quad \text { for some } \delta>0 \text { and } \quad v_{0}=\frac{\varrho d}{1+\varrho d} E(v),
$$

is a Bernoulli-shift.

Proof. By Lemma (5.5) it is enough to show that the partition $\eta^{(k)}$ is weakly-Bernoulli under $S$.

Let $\varepsilon>0$ be given. Take $t_{1}=t_{1}(\varepsilon, \delta)$ as defined in Lemma (6.2) and let $\bar{\zeta}=\zeta \vee\left\{G_{t_{1}}, G_{t_{1}}^{c}\right\}$.

Because of the $K$-property of our system $\exists t_{2}(\varepsilon)>0$ such that $\bar{\zeta}$ is $\frac{\varepsilon}{10}$-independent of

$$
\bigvee_{i=-\left(t_{1}+t_{2}+m\right)}^{-\left(t_{1}+t_{2}+1\right)} S^{i} \eta^{(k)}, \quad \forall m \geqq 1^{3}
$$

We now claim that $\forall m \geqq 1$, the partition

$$
\bigvee_{t_{1}}^{t_{1}+m} S^{i} \eta^{(k)}=\left\{a_{l}^{(m)}\right\}
$$

3 This is so (see [6]) because the partitions $\eta^{(k)}$ all have finite entropy (since the velocity distribution $v$ has a finite first moment). We wish to point out, however, that the argument which we give does not depend in an essential way upon the finiteness of the entropy of $\eta^{(k)}$. We could easily find an increasing sequence $\eta_{l}^{(k)} \uparrow \eta^{(k)}$ of finite partitions whose supremum is $\eta^{(k)}$; our argument could be applied exactly as it stands to the $\eta_{l}^{(k)}$, so that Theorem (6.6) would be valid with $\eta_{l}^{(k)}$ in place of $\eta^{(k)}$. Theorem (6.6) would then, itself, follow from an application of Lemma (5.2). 
is $\varepsilon$-independent of

$$
\bigvee_{i=-\left(t_{1}+t_{2}+m\right)}^{-\left(t_{1}+t_{2}+1\right)} S^{i} \eta^{(k)}=\left\{b_{j}^{(m)}\right\}
$$

To see this, note that by virtue of Lemma (6.5) (omitting the superscript $m$ )

$$
\mu\left(a_{l} \mid b_{j}, \bar{\zeta}_{k}\right)=\mu\left(a_{l} \mid \bar{\zeta}_{k}\right)
$$

$\forall j, l$ and $\bar{\zeta}_{k} \subset G_{t_{1}}$ an element of $\bar{\zeta}$.

This implies that

Therefore

$$
\begin{aligned}
\mu\left(a_{l} \mid b_{j}\right)= & \sum_{\zeta_{k} \subset G_{t_{1}}} \mu\left(a_{l} \mid \bar{\zeta}_{k}\right) \cdot \mu\left(\bar{\zeta}_{k} \mid b_{j}\right) \\
& +\mu\left(a_{l} \mid b_{j}, G_{t_{1}}^{c}\right) \cdot \mu\left(G_{t_{1}}^{c} \mid b_{j}\right) .
\end{aligned}
$$

$$
\begin{aligned}
\sum_{l}\left|\mu\left(a_{l} \mid b_{j}\right)-\mu\left(a_{l}\right)\right| \leqq & \sum_{\zeta_{k} \subset G_{t_{1}}}\left|\mu\left(\bar{\zeta}_{k} \mid b_{j}\right)-\mu\left(\bar{\zeta}_{k}\right)\right| \\
& +\mu\left(G_{t_{1}}^{c} \mid b_{j}\right)+\mu\left(G_{t_{1}}^{c}\right) .
\end{aligned}
$$

Now, 1) the above sum is smaller than $\frac{\varepsilon}{10}$ except possibly for sets $b_{j}$ whose total measure is smaller than $\frac{\varepsilon}{10}$,

2) $\mu\left(G_{t_{1}}^{c}\right)<\left(\frac{\varepsilon}{10}\right)^{2}$

3) 2) implies that $\mu\left(G_{t_{1}}^{c} \mid b_{j}\right)<\frac{\varepsilon}{10}$, except for a collection of sets whose total measure is less than $\frac{\varepsilon}{10}$. Summing the above estimates we obtain

$$
\sum_{l}\left|\mu\left(a_{l}^{(m)} \mid b_{j}^{(m)}\right)-\mu\left(a_{l}^{m}\right)\right|<\varepsilon
$$

for a collection of elements $b_{j}^{(m)}$ whose total measure exceeds $1-\varepsilon$, proving the claim.

Since $t_{1}(\varepsilon), t_{2}(\varepsilon)$ are independent of $m, \eta^{(k)}$ is weakly-Bernoulli.

In order to apply Theorem (6.6) to the proof of (6.1) we need the following lemma

Lemma (6.7). $\eta$ is a generating partition.

Proof. Observe that knowing $N(t, y)$ and the characteristics of a pulse in $S^{t} y$ enables one to find the (reduced) position of the pulse in $y \in Y$. Since, by Lemma (2.1), each pulse is marked at some time (with probability 1) $\bigvee_{i=-\infty}^{\infty} S^{i} \eta$ separates points in $Y(\bmod 0)$. 
Proof of Theorem (6.1).

By the previous lemma, the processes considered in Theorem (6.6) have the property required for application of Lemma (5.2), by which (6.1) follows. Q.E.D.

\section{Conclusion}

In summary, the essential ideas of the proof are:

1) The infinite hard rod system admits a representation by generating $K$-process obtained by observing the pulses close to the origin (in the reduced description, for convenience).

2) Rods which cross the origin tend to draw back pulses which crossed before them. $d N(t, \cdot) / t$ is the random velocity with which pulses are "pursued" by the origin (this has a clearer meaning in the reduced description).

3) Due to the random character of the above velocity (which tends to $v_{0}$ in the limit $\left.t \rightarrow \pm \infty\right), \forall \delta, \varepsilon>0 \exists t>0$ for which, with probability $>1-\varepsilon$, no pulse which appeared at the origin before $-t$ could reappear after the time $t$ with the exception of those whose velocity is $\delta$ close to $v_{0}$.

4) When viewed as a process, systems from which pulses slow relative to $v_{0}$ are excluded exhibit an approximate Markov property.

5) A generating family of processes, which are constructed on countable partitions and have an approximate Markov property, can be found. The Bernoulli property for these is proved in a way similar to the proof for a Markov, $K$-process.

Acknowledgements. We would like to thank Oscar E. Lanford III and Oliver Penrose for valuable discussions.

\section{Appendix: A Clarification of the Proof of Sinai's Result}

Sinai [1] constructed a $K$-partition ( $\zeta_{0}$ in his notation) for a one dimensional hard rod gas with an infinite number of degrees of freedom. However, Remark 4 in his article is incorrect as stated, leaving the proof of the generating property of the partition incomplete.

$t$ is called there a moment of intersection of zero of the trajectory of point $x \in X$, if either

1) $x_{i}(t)=0$ for some $i$ (there is a pulse in $S_{t} x$ which is crossing the origin), or

2) $t$ is a moment of collision of two rods which at this time are on opposite sides of the origin (and therefore two pulses in $S_{t} x$ are jumping across the origin). 
The intersection time, velocities and positions of the pulses involved are called the characteristics of the intersection.

One still has to show that the generated partition $\left(\bigvee_{t} S_{t} \zeta_{0}\right)$, which corresponds to partitioning by the characteristics of all the intersections, completely separates the set of phase space points for which any pulse has a moment of intersection of the origin (which was shown to be a subset of full measure). This can be done using the reduced description.

The reduced position of a pulse in $x$ can be obtained from its reduced position in $S_{t} x$ by

$$
q(o)=q(t)-t v+d N(t, x) .
$$

Further, by looking for the first, in terms of lowest $|t|$, intersection of zero of a pulse in $x$ one can determine whether its index is positive or negative. In this method, provided each pulse eventually crosses the origin, one can reconstruct the reduced description of $x$ from the characteristics of all the intersections, which therefore determine it uniquely $(\bmod 0)$.

\title{
References
}

1. Sinai, Y. G. : Funct. Anal. Appl. 6, 35 (1972)

2. Goldstein, S., Lebowitz, J. L.: Commun. math. Phys. 37, 1-18 (1974)

3. Ruelle, D.: Statistical mechanics-rigorous results. New York: Benjamin 1969

4. Goldstein, S. : Space-time ergodic properties of systems of infinitely many independent particles. Commun. math. Phys. (in press) (1974)

5. Ornstein,D.S.: Ergodic theory, randomness, and dynamical systems. Lecture notes from Stanford University

6. Smorodinsky, M.: Ergodic theory, entropy, Springer Lecture Notes 214 (1970)

7. Shields, P.: The theory of Bernoulli shifts. University of Chicago Press

Communicated by G. Gallavotti

\author{
Michael Aizenman \\ Joel L. Lebowitz \\ Belfer Graduate School of Science \\ Yeshiva University \\ New York, N.Y. 10033, USA \\ Sheldon Goldstein \\ Institute for Advanced Study \\ Princeton, New Jersey 08540, USA
}


